\title{
Modulation of Proprioceptive Integration in the Motor Cortex Shapes Human Motor Learning
}

\author{
Karin Rosenkranz and John C. Rothwell \\ Sobell Department of Motor Neuroscience and Movement Disorders, University College London Institute of Neurology, London WC1N 3BG, \\ United Kingdom
}

\begin{abstract}
Sensory and motor systems interact closely during movement performance. Furthermore, proprioceptive feedback from ongoing movements provides an important input for successful learning of a new motor skill. Here, we show in humans that attention to proprioceptive input during a purely sensory task can influence subsequent learning of a novel motor task. We applied low-amplitude vibration to the abductor pollicis brevis (APB) muscle of eight healthy volunteers for $15 \mathrm{~min}$ while they discriminated either a small change in vibration frequency or the presence of a simultaneous weak cutaneous stimulus. Before and after the sensory attention tasks, we evaluated the following in separate experiments: (1) sensorimotor interaction in the motor cortex by testing the efficacy of proprioceptive input to reduce $\mathrm{GABA}_{\mathrm{A}}$ ergic intracortical inhibition using paired-pulse transcranial magnetic stimulation, and (2) how well the same subjects learned a ballistic thumb abduction task using the APB muscle. Performance of the vibration discrimination task increased the interaction of proprioceptive input with motor cortex excitability in the APB muscle, whereas performance in the cutaneous discrimination task had the opposite effect. There was a significant correlation between the integration of proprioceptive input in the motor cortex and the motor learning gain: increasing the integration of proprioceptive input from the APB increased the rate of motor learning and reduced performance variability, while decreasing proprioceptive integration had opposite effects. These findings suggest that the sensory attention tasks transiently change how proprioceptive input is integrated into the motor cortex and that these sensory changes drive subsequent learning behavior in the human motor cortex.
\end{abstract}

\section{Introduction}

The sensory and motor cortices are shaped by experience and learning (Jenkins et al., 1990; Monfils et al., 2005; Xerri, 2008). It is well known that motor learning engages the motor cortex directly (Rioult-Pedotti et al., 1998; Muellbacher et al., 2002) and also relies on reafferent sensory feedback, particularly proprioception, for optimization (Pavlides et al., 1993). Interestingly, motor learning also impacts on the sensory system by changing sensory function, especially proprioception (Ostry et al., 2010; Wong et al., 2011). However, it is not known whether the converse it true: that is, do interventions that change the way sensory input is processed influence motor learning? In an earlier study in humans, we showed that learning a ballistic thumb abduction task facilitates motorcortical integration of proprioceptive input from the muscle that is most engaged in this task, the abductor pollicis brevis (APB) (Rosenkranz and Rothwell, 2006a). Here, we ask whether this works also in reverse, that is, whether mod-

\footnotetext{
Received 0ct. 6, 2011; revised April 23, 2012; accepted May 15, 2012.

Author contributions: K.R. and J.C.R. designed research; K.R. performed research; K.R. analyzed data; K.R. and J.C.R. wrote the paper.

This work was supported by the Dystonia Medical Research Foundation. We thank Dr. Aleksandra Kacar (Clinical Center Serbia, Institute of Neurology, Belgrade, Serbia) for assistance in the experiments, and Danilo Mandic (Imperial College London, London, UK) for helpful discussion and comments on this manuscript.

The authors declare no competing financial interests.

Correspondence should be addressed to Dr. Karin Rosenkranz, Department of Cognitive and Clinical Neuroscience, Central Institute of Mental Health, Medical Faculty Mannheim, University of Heidelberg, Square J 5, 68159 Mannheim, Germany. E-mail: karin.rosenkranz@zi-mannheim.de.

DOI:10.1523/JNEUROSCI.0120-12.2012

Copyright $\odot 2012$ the authors $\quad 0270-6474 / 12 / 329000-07 \$ 15.00 / 0$
}

ulating the motorcortical integration of proprioceptive input from the APB before motor learning influences the learning process.

Integration of proprioceptive input in the motor cortex can be measured using a paired-pulse transcranial magnetic stimulation (TMS) paradigm to test $\mathrm{GABA}_{\mathrm{A}}$ ergic intracortical inhibition (Kujirai et al., 1993; Ziemann et al., 1996; Ilić et al., 2002; Rosenkranz and Rothwell, 2003; Di Lazzaro et al., 2006; Florian et al., 2008). This is performed either alone or during muscle spindle Ia-afferent input evoked from the resting target muscle by lowamplitude vibration (Burke et al., 1976; Roll et al., 1989). Vibration reduces intracortical inhibition of corticospinal outputs to the vibrated muscles, while it increases inhibition to surrounding hand muscles. This pattern of vibration-sensitive GABAergic inhibition is termed "sensorimotor organization" (SMO) (for methodological details, see Rosenkranz and Rothwell, 2003).

SMO can be changed temporarily if volunteers perform shortlasting (15 min) sensory attention tasks during discontinuous hand muscle vibration (Rosenkranz and Rothwell, 2006b). The present experiments use two versions of these tasks with vibration applied to the APB. Eight subjects were asked either to discriminate subtle changes in the vibration frequency (AttVIB) or to detect faint electrical cutaneous stimuli applied to the skin overlying the muscle (AttCUT) (for details, see Materials and Methods). On the basis of our earlier findings, we expected these sensory attention tasks to modulate temporarily and differentially the motorcortical integration of proprioceptive input from the APB. 
In separate sets of experiments, we tested the effect of AttVIB and AttCUT on motor learning in the same subjects. The motor learning task directly engaged the APB muscle as it required subjects to maximize peak acceleration of ballistic thumb abductions (Muellbacher et al., 2002; Rosenkranz et al., 2007). Neurophysiological and behavioral effects of the sensory attention tasks were correlated to test for relationships in each subject between modulation of proprioceptive integration and learning behavior.

\section{Materials and Methods}

Subjects. Eight healthy, right-handed subjects (four females), aged $35.6 \pm$ 2.8 years, gave informed consent for the study, which was approved by the local ethics committee and conformed to the Declaration of Helsinki.

SMO and TMS. The measurement of SMO followed previously described protocols (Rosenkranz and Rothwell, 2003, 2006a,b; Rosenkranz et al., 2007) using single- and paired-pulse TMS. TMS was performed using two MAGSTIM 200 stimulators connected to a figure-of-eightshaped coil ( $7 \mathrm{~cm}$ internal wing diameter) by a Y-cable (Magstim). The coil was held with the handle pointing backward and laterally $\sim 45^{\circ}$ to the interhemispheric line to evoke anteriorly directed current in the brain and was optimally positioned to obtain motor evoked potentials (MEPs) in the APB muscle. Single TMS pulses (test pulse alone) or pairs of pulses (conditioning and test pulse; interstimulus interval, $3 \mathrm{~ms}$ ) (Kujirai et al., 1993) to measure the short-interval intracortical inhibition (SICI) were applied randomly every $5 \mathrm{~s}$. The intensity of the test stimulus was set to evoke an MEP of $1 \mathrm{mV}$ peak-to-peak amplitude, and the subthreshold conditioning stimulus was set to evoke a 50\% inhibition of the test MEP, which approximated an intensity of $80 \%$ of active motor threshold. On one-third of trials each, stimuli were applied either in the presence of APB vibration (vibAPB) or FDI vibration (vibFDI) or without vibration, in a randomized order.

The vibration was of low amplitude $(0.2-0.5 \mathrm{~mm}$; individually adapted to be well below the threshold for perceiving illusory movements) and was applied in trains of $1.5 \mathrm{~s}$ duration (followed by $3.5 \mathrm{~s}$ vibration-free interval) to the muscle belly of either the APB (vibAPB) or FDI (vibFDI) muscles (frequency of $80 \mathrm{~Hz}$; amplitude, 0.2-0.5 mm;) using $0.7 \mathrm{~cm}$ diameter probes each connected to an electromagnetic stimulator (Ling Dynamics System). We monitored the electromyogram (EMG) in the vibrated muscle continuously for any signs of muscle contraction that might reflect either a possible voluntary movement or the occurrence of the tonic vibration reflex (Hagbarth and Eklund, 1968; Marsden et al., 1969). However, the low-amplitude of the vibration neither induced an artifact in the EMG trace nor an illusory movement sensation. The TMS single pulse or test pulse was applied every $5 \mathrm{~s}$ and was timed to appear $1 \mathrm{~s}$ after the onset of vibration. A total of 60 trials were collected with 10 trials of each condition.

Surface EMG recordings in a belly-to-tendon montage were made from the APB, the first dorsal interosseus (FDI) and the abductor digiti minimi (ADM) muscle of the right hand. The raw signal was amplified and bandpass filtered between $30 \mathrm{~Hz}$ and $1 \mathrm{kHz}$ (Digitimer). Signals were digitized at $2 \mathrm{kHz}$ (CED Power1401; Cambridge Electronic Design) and stored on a laboratory computer for off-line analysis.

Sensory attention tasks. The AttVIB and AttCUT tasks [adapted from the study by Rosenkranz and Rothwell (2006b)] both consisted of $15 \mathrm{~min}$ of repeated cycles ( $2 \mathrm{~s}$ on, $2 \mathrm{~s}$ off) of vibration applied to the APB muscle of the right hand (vibration parameter same as for SMO) (see above) but differed in the concurrent discrimination task in terms of sensory modality-specific attention. In AttVIB, vibration frequency was changed in $70 \%$ of trials at random from $80 \mathrm{~Hz}$ to $65,67.5,70,72.5,75$, or $77.5 \mathrm{~Hz}$ for the last $300 \mathrm{~ms}$ of the train. In the $2 \mathrm{~s}$ rest period, subjects had to report whether they perceived a frequency change by pressing buttons on a response box with their left hand. The subjects got auditory feedback when their response was correct. In AttCUT, subtle electrical stimuli (just above perceptual threshold) were applied to the skin overlying the APB muscle belly at the base of the thenar eminence. These stimuli were given randomly within the $4 \mathrm{~s}$ cycle ( 2 s vibration on, 2 s vibration off). Subjects had to press a button with their left hand when they perceived the stimulus and were given auditory feedback when their reaction occurred

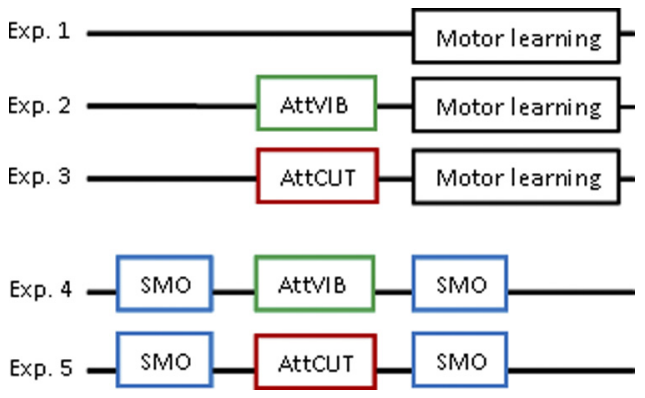

Figure 1. Experimental design. Each of the subjects took part in five different experiments, which were separated by several weeks $(>2)$. Experiments $1-3$ were behavioral experiments that tested motor learning with or without preceding sensory attention task. Experiments 4 and 5 were neurophysiological experiments that tested the change of SMO as induced by the sensory attention tasks. The order of experiments was randomized for each subject.

within a time window of $300 \mathrm{~ms}$ after the cutaneous stimulus. AttCUT was performed as a simple reaction time task to match the level of attentional demand to the AttVIB condition, to give the subjects feedback about their performance and to keep them motivated to perform as well as possible.

In AttVIB and AttCUT, the spatial location of subjects' attention was similar while the attended sensory modality was varied. Both sensory attention tasks required subjects to make the same number of discriminations $(N=225)$ and were comparable in their attentional demand.

Motor learning task. The motor learning task followed previously described protocols (Rosenkranz and Rothwell, 2006a; Rosenkranz et al., 2007). Subjects were asked to make rapid abduction movements with their right thumb and, for doing so, to activate their APB as selectively as possible. The movements were paced by a brief $1000 \mathrm{~Hz}$ tone at a rate of $0.5 \mathrm{~Hz}$. Performance was monitored on-line by the experimenter. The subjects performed two blocks of $4 \mathrm{~min}$ each, separated by a $4 \mathrm{~min}$ break to prevent fatigue. Thumb acceleration was measured using a uniaxial accelerometer (Vibro-Meter SA) attached to the distal phalanx in the abduction-adduction axis. The raw signal was amplified and digitized (A/D rate, $2 \mathrm{kHz}$; CED Power1401) and fed into the computer for off-line analysis. Subjects received on-line visual feedback during the task and were continuously encouraged to improve their performance. The performance was quantified by two performance measures: (1) the mean peak acceleration, as averaged for each set of 30 movements (i.e., per minute training), which gives a direct measure of the performance level per minute training; and (2) the coefficient of variance (CoVar), calculated over each set of 30 movements, which gives a measure of the performance variability per minute training.

Experimental design. Each of the subjects took part in two neurophysiological and three behavioral experimental sessions. The order of sessions was randomized and the single sessions were separated by several weeks ( $>2$ weeks) to avoid potential culmination of learning effects. In the two neurophysiological experiments, the effect of the two different sensory attention tasks on SMO of the hand motor area was tested. In the three behavioral experiments, motor learning of a ballistic thumb abduction task was tested without preceding sensory attention tasks (baseline) and either with preceding AttVIB or AttCUT task (Fig. 1).

Data analysis and statistics. TMS parameters (active motor threshold, stimulation intensities of conditioning and test pulses) used in the two neurophysiological experiments were compared for each subject using paired $t$ tests and no significant differences were found. Furthermore, between the three behavioral experiments the comparability of the mean thumb acceleration and CoVar in the first minute of motor training was confirmed by calculating a one-way ANOVA, which showed no significant difference. For the performance data of the sensory attention tasks, the mean error (AttVIB) per minute training and mean reaction time (AttCUT) per discrimination condition (timing of cutaneous stimulus in relation to vibration) were calculated. Repeated-measures ANOVAs were used to analyze the neurophysiological data (SMO) using the factors "muscle," "vibration condition," and "intervention," and to analyze 
A

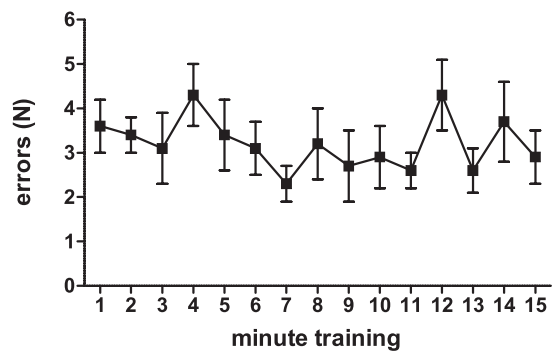

B

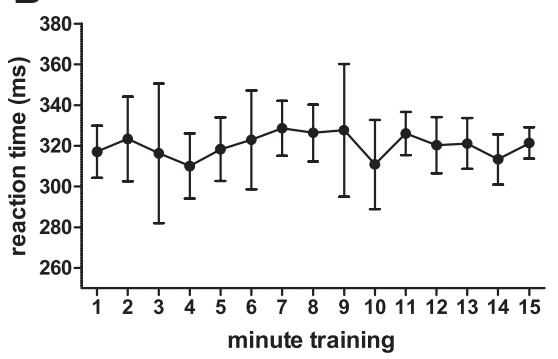

Figure 2. Behavioral data of the sensory attention tasks. Group average of the behavioral data of the sensory attention tasks. $\boldsymbol{A}$ shows the mean error per minute training ( \pm SEM) for $A t t V I B$, and $B$ shows the mean reaction time per minute ( \pm SEM) for AttCUT. The performance did not change significantly in either of the sensory attention tasks over the $15 \mathrm{~min}$ period.

Table 1. TMS parameter (percentage stimulator output \pm SEM)

\begin{tabular}{llll}
\hline & aMT & Cond. SI & Test SI \\
\hline AttVIB & $31.3 \pm 2.5$ & $26.0 \pm 1.6$ & $50.8 \pm 4.3$ \\
AttCUT & $31.0 \pm 2.3$ & $25.9 \pm 1.8$ & $49.6 \pm 3.8$ \\
$t$ test & NS & NS & NS \\
\hline
\end{tabular}

SI, Stimulus intensity; cond., conditioning.

the motor learning data (mean peak acceleration/minute; CoVar/minute) using the factors "intervention" and "minute of training." In addition, repeated-measures ANOVAs were used to analyze the sensory attention task performance data separately for AttVib and AttCUT using the factor minute of training.

To calculate the correlation of intervention-induced changes of SMO and motor learning, both data sets were simplified. For the SMO, the differences (after - before intervention) of the SICI obtained during vibAPB were calculated and correlated with the motor learning data, approximated as the slopes of the linear regression through the given data points of the motor learning curve as given in Figure 2. Figure 3 shows the $r^{2}$ values for significant correlations.

Significance levels for the ANOVAs are set to $p<0.01$ to correct for multiple comparisons, and for the $t$ tests to $p<0.05$. The GreenhouseGeisser test was used to check for sphericity of the data. All data are given as means \pm SEM.

\section{Results}

The TMS parameters (active motor threshold, stimulation intensities of conditioning, and test pulses) used in the two neurophysiological experiments were not significantly different (Table 1); furthermore, between the three behavioral experiments, the mean thumb acceleration and CoVar in the first minute of motor training were not significantly different $\left(F_{(2,14)}<1 ; p>0.4\right)$.

Figure 1 shows the results of the discrimination tasks during AttVIB and AttCUT as errors (for AttVIB) and mean reaction time (for AttCUT) per minute training. There was no significant difference in the performance data over the time of training $\left(F_{(2,14)}<1.3 ; p>0.2\right)$.
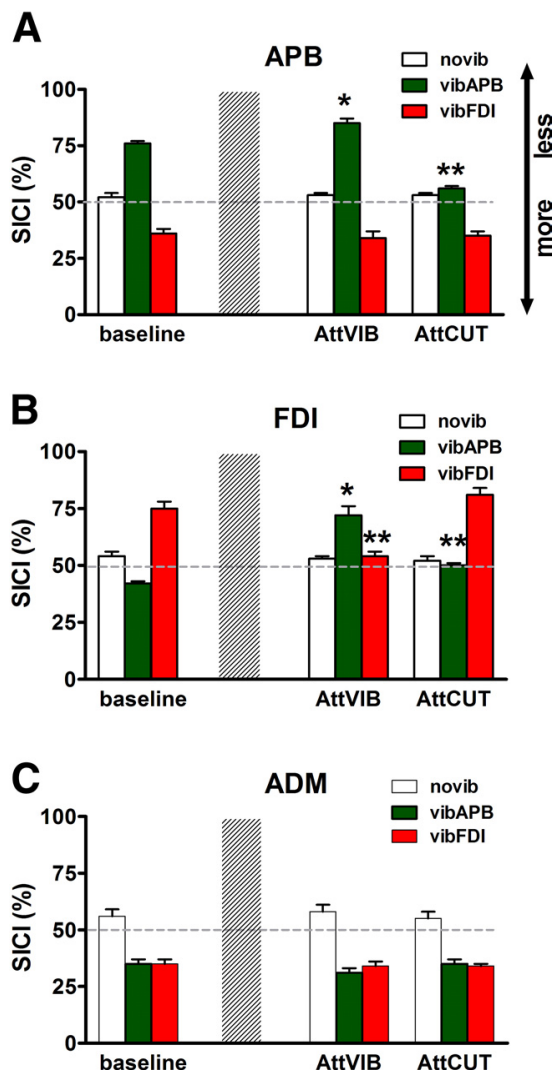

Figure 3. SMO before (baseline) and after the AttVIB and AttCUT tasks. The three graphs plot the SICI measured without vibration (novib) and with vibration of either APB (vibAPB) or FDI (vibFDI) in the APB (A), FDI (B), and ADM (C). SICl data are shown as percentage conditioned/ test MEP ( $\pm S E M)$. The two baseline SMO were pooled for purpose of display. At baseline, $\mathrm{SICl}$ is reduced (columns going up) in the vibrated muscle, and increased (columns going down) in the nonvibrated muscles. After AttVIB, vibAPB induced an even stronger reduction of SICl in APB, and now reduced SICl in FDI, too. After AttCUT, the effect of vibAPB on APB and FDI was abolished. Neither intervention had an effect on the SMO recorded in ADM, probably because its cortical representation is functionally more separated from the APB and FDI. The asterisks indicate significant differences from corresponding baseline values (paired $t$ tests; ${ }^{*} p<0.005$; $\left.{ }^{* *} p<0.0001\right)$.

In the two neurophysiological experiments (Fig. 3), the effect of the sensory attention tasks on SMO was measured to obtain data for correlation analyses with the behavioral results. As in previous studies on healthy subjects (Rosenkranz and Rothwell, 2003, 2006a,b), at baseline, SMO was organized such that vibration reduced SICI in the vibrated muscle and increased SICI in the nonvibrated hand muscles $\left(F_{(2,14)}>304.94 ; p<0.0001\right)$. After AttVIB, the pattern of SMO was changed in two ways: first, proprioceptive input from the APB produced a stronger reduction of SICI in the APB than at baseline, and second, it reduced (rather than increased) SICI in projections to the nonvibrated FDI muscle $\left(F_{(2,14)}=77.8 ; p<0.0001\right)$. After AttCUT vibration of the APB had no modulatory effect on SICI measured in the $\mathrm{APB}$ and FDI $\left(F_{(2,14)}=77.8 ; p<0.0001\right)$. None of the sensory attention tasks changed the effect of APB or FDI vibration on SICI measured in the ADM, which shows that their effects are relatively focused.

It is important to note that SICI measured without vibration was unchanged by the AttVIB and AttCUT interventions; similarly, the MEPs (data not shown) measured after a single TMS pulse were unchanged (mean MEP baseline, $0.94 \pm 0.24 \mathrm{mV}$; after AttVIB, $0.97 \mathrm{mV} \pm$ 0.22; after AttCUT, $0.95 \pm 0.20 \mathrm{mV}$ ). Therefore, the general level of motor excitability (as measured 
A

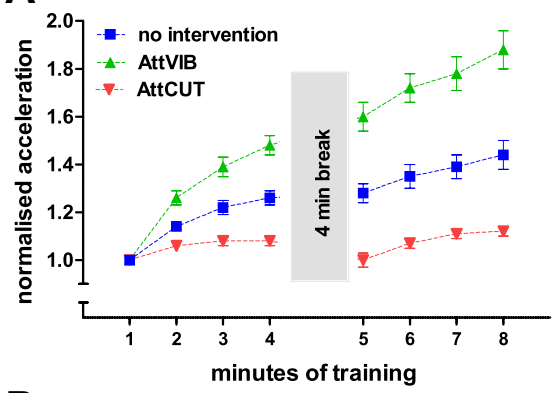

B

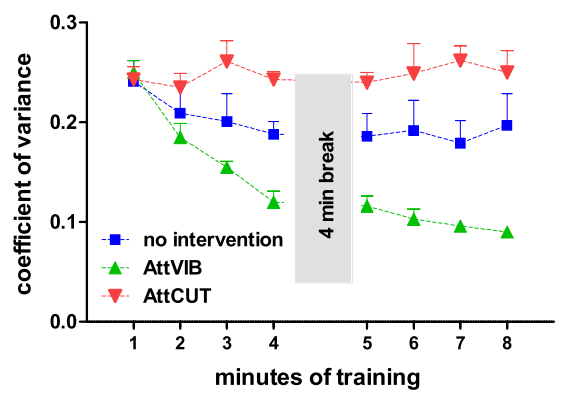

Figure 4. Motor learning. The mean peak acceleration $(\boldsymbol{A})$ normalized relative to the mean acceleration during the first minute of training ( \pm SEM), and the mean CoVar ( \pm SEM) $(B)$ for the thumb abduction movement per minute of training are shown for the three experiments, without (no intervention) or with either preceding AttVIB or AttCUT. The mean acceleration and CoVar during the first minute of training were not significantly different between the experiments $(p=0.56) . A$, In the "no-intervention" condition, the mean acceleration increased over the training time $(p<0.00001)$, indicating a learning effect. This was significantly enhanced by the preceding AttVIB task and significantly decreased by the AttCUT task $(p<0.0001)$. $\boldsymbol{B}$, After AttVIB, the variability of performance was significantly reduced with training compared with the condition without preceding intervention $(p<0.001)$, whereas after AttCUT the performance variability did not change.

without vibration) remained unchanged, while the sensory attention tasks appear to have specifically altered the effect of proprioceptive input from APB on SICI.

In the three behavioral experiments, motor learning of a ballistic thumb abduction task was tested without (baseline condition) and with preceding AttVIB or AttCUT (Fig. 4). In the baseline condition, subjects learned to improve performance, as shown by the gradual increase of acceleration during the two 4 min training blocks $\left(F_{(7,49)}=38.7 ; p<0.00001\right)$; furthermore, the variability of performance of the thumb abductions decreased slightly (coefficient of variation). When the motor learning task was preceded by AttVIB, the increase in acceleration was much steeper than at baseline $\left(F_{(7,49)}=31.4 ; p<0.0001\right)$; this was accompanied by a significant decrease of performance variability $\left(F_{(7,49)}=34.58 ; p<0.001\right)$. The opposite happened when AttCUT preceded motor learning; here, acceleration increased significantly slower than at baseline $\left(F_{(7,49)}=20.2 ; p<0.0001\right)$ and the variability of performance did not change with learning. The mean acceleration in the first minute of motor training was similar in all three experiments independent of the order in which they were performed $\left(F_{(2,14)}=2.41 ; p=0.56\right)$, which excludes a culmination or retention of skills over different sessions.

These results show that the preceding sensory attention tasks changed the rate of motor learning. Importantly, the direction of the effects was determined by the stimulus modality that volunteers had to discriminate during the sensory attention task $\left(F_{(14,98)}=42.1 ; p<0.0001\right)$ : motor learning was facilitated by AttVIB, the intervention that required subjects to focus on pro-
A
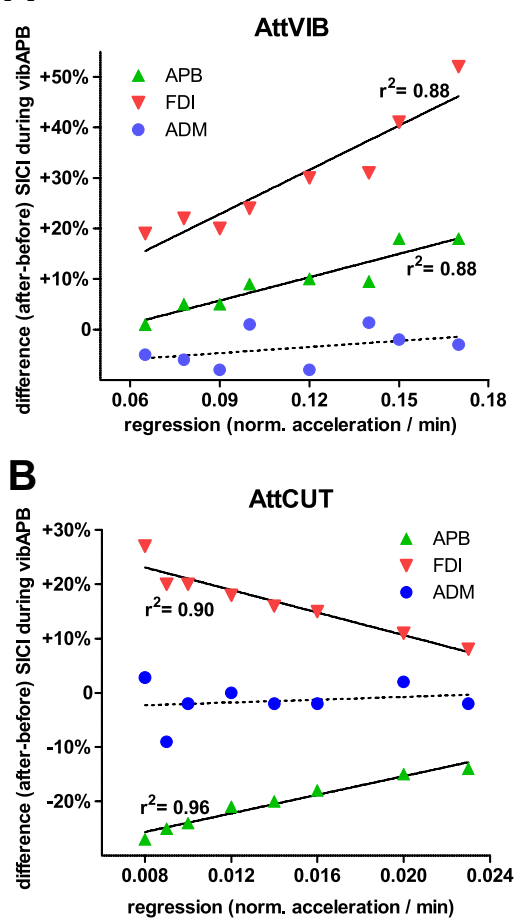

Figure 5. Behavioral-neurophysiological correlation. The two graphs plot the correlation of the peak thumb acceleration and the change to SMO induced by the AttVIB $(\boldsymbol{A})$ and AttCUT (B) tasks. The linear regression is calculated on the mean peak acceleration data per minute during thumb abduction training ( $x$-axis) versus the difference of SICI (after - before intervention) recorded during vibration of APB in all three hand muscles ( $y$-axis). Both sensory attention tasks induced a correlated effect on SMO and motor learning. After AttVIB, the more APB vibration reduced SICI in APB and FDI (increase in percentage conditioned/test MEP: less SICI), the faster was the increase in thumb acceleration. After AttCUT, the more SICI increased in APB (decrease in percentage conditioned/test MEP: stronger SICI) and decreased in FDI, the slower was the increase of acceleration. For the significant correlations, $r^{2}$ is given.

prioception; conversely, motor learning was reduced or almost abolished by AttCUT, the intervention that required subjects to focus on cutaneous stimulation and to ignore proprioception.

In the neurophysiological and behavioral experimental sessions, the subjects' error rate (error/minute) in discriminating the vibration frequency (AttVIB) and detecting the cutaneous stimulation (AttCUT) were not different between tasks nor experimental sessions $\left(F_{(28,120)}<0.8 ; p>0.1\right)$ and were not correlated to changes in either SMO or motor learning $(p>0.3)$.

To test whether the neurophysiological and behavioral changes induced by the sensory attention tasks were interrelated, we simplified the SMO and motor learning data sets and performed a correlation analysis (Fig. 5). The SMO changes (after before sensory attention tasks) were summarized as the effect of APB vibration on the SICI, since the APB was vibrated in AttVIB and AttCUT and was the muscle most engaged in the motor learning task. The gain of motor learning was calculated as the slope of the linear regression calculated on the normalized mean acceleration per minute as given in Figure 4. After AttVIB, subsequent APB vibration reduced SICI in the APB significantly more than at baseline; furthermore, it now reduced, rather than increased, SICI in FDI. The motor learning gain was significantly $(p<0.008)$ correlated to the decrease of SICI in APB (Pearson's $r=0.89)$, as well as to the novel decrease of SICI in FDI (Pearson's $r=0.83$ ): the more APB vibration reduced SICI in $\mathrm{APB}$ and FDI, the faster was the increase in acceleration. In 

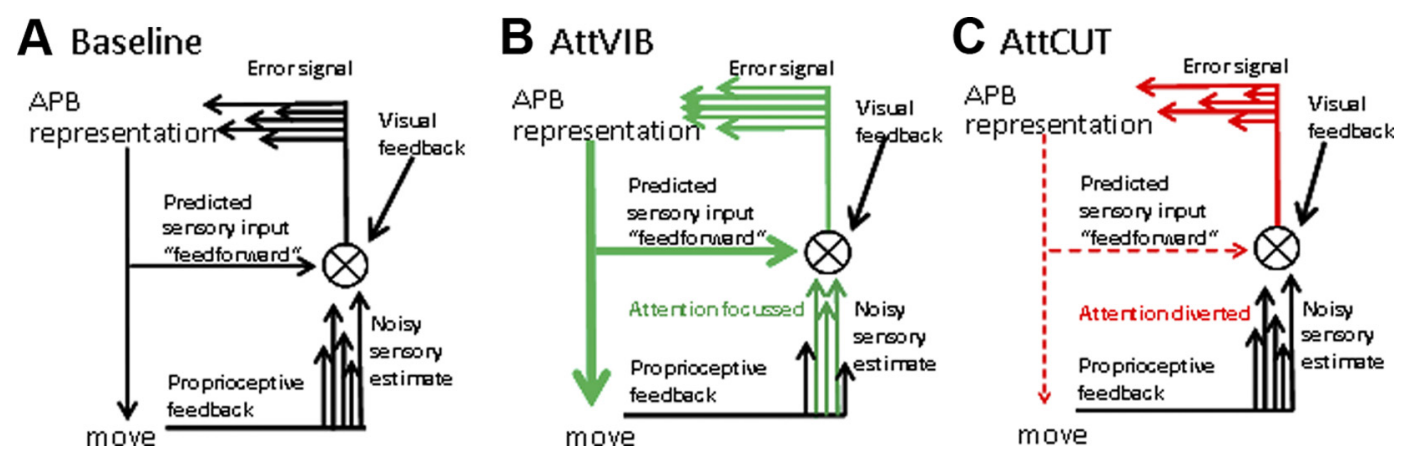

Figure 6. Model: modulations of proprioceptive-motor integration influence subsequent motor learning. $A$, Abduction movements of the thumb evoke proprioceptive movement feedback from the APB muscle. This feedback signal is intrinsically noisy so that, when compared with the position predicted on the basis of efferent copy, the "error" signal is equally noisy. This estimate of error is then used to optimize the motor command for the next movement. Visual feedback of the peak acceleration is provided to the subjects and contributes to this optimization process. $\boldsymbol{B}$, AttVIB directs the attentional focus of the subjects on proprioceptive input from the APB, potentially by top-down controlled sensory gating processes. Thus, at the start of the motor learning, the noise of the sensory estimate is reduced. Consequently, during learning, proprioceptive input gains easier access to the motor cortex since the variability of the error signal is reduced, which consequently leads to a higher learning gain. $C$, AttCUT directs subjects' attention away from proprioceptive input. Consequently, during subsequent learning, the sensory estimate and the error signal are not easily modified, which prevents motor learning.

addition, the increased learning gain after AttVIB was strongly correlated to a reduced performance variability (Pearson's $r=$ $-0.96 ; p<0.001)$.

After AttCUT, subsequent APB vibration lost its modulating effect on SICI in APB and FDI. In both muscles the SICI during APB vibration became similar to levels recorded without vibration, meaning that the SICI during APB vibration increased in $\mathrm{APB}$, while it decreased in FDI. Both changes were significantly $(p<0.0007)$ correlated to the gain of motor learning: the more SICI increased in APB (Pearson's $r=0.82$ ) and decreased in FDI (Pearson's $r=-0.91$ ), the slower was the increase of acceleration. There was no correlation of the mean acceleration and the performance variability over the training period.

\section{Discussion}

The present study shows for the first time that two sensory attention tasks that modulate proprioceptive integration (SMO) in the motor cortex can influence subsequent motor learning in humans. Indeed, the effect on learning correlated with the degree of change in SMO. We suggest that the sensory attention tasks specifically modulated the influence of proprioceptive input on GABA-dependent inhibition in the motor cortex. The result of this was that the AttVIB task focally increased the integration of reafferent proprioceptive feedback and boosted subsequent motor learning, whereas the AttCUT task had the opposite effect and reduced learning.

\section{SMO and attention tasks}

Circuits involved in SICI are thought to be highly important in motor control and motor learning. The fact that proprioceptive input evoked by muscle vibration reduces SICI in the vibrated hand muscle and increases it in the nonvibrated hand muscles is an indication of spatially specific proprioceptive-motor integration (Rosenkranz and Rothwell, 2003). Similar effects could not be evoked by using, for example, cutaneous stimulation (Rosenkranz and Rothwell, 2003), which is probably due to the fact that proprioceptive input is not only integrated in area 3a but also directly in area 4 of the sensorimotor cortex (Heath et al., 1976; Hore et al., 1976; Fromm et al., 1984; Huffman and Krubitzer, 2001; Golaszewski et al., 2002; Burton et al., 2008).

The sensory attention tasks changed the effectiveness of this proprioceptive-motor integration. Both of them required subjects to focus their attention on the same spatial location, the thenar emi- nence of their right hand, and were similarly demanding. It is therefore unlikely that differences in their effects on SMO and motor learning are due to differences in either spatial focus or amount of subjects' attention. Instead, we suggest that the effect is specific for the sensory modality of the attended stimulation (proprioceptive vs cutaneous) and that attention exerted a top-down control on sensory integration by modality-specific gating, which selectively facilitated (in case of AttVIB) or reduced (in case of AttCUT) the central integration of proprioceptive input (Alitto and Usrey, 2003).

\section{Motor learning in the thumb abduction task}

This task requires participants to optimize activity of muscles controlling the thumb to maximize initial acceleration as indicated on a visual display. Agonist and synergist muscles must be activated simultaneously and maximally, while antagonists must be silenced. Once the skill is learned, it is remembered; performance 1 week later is still better than at baseline.

Thumb abduction learning can be viewed, at least in its initial stages, as type of model-free learning in which successful (i.e., faster) movements are remembered at the expense of poor (slow) movements. It requires that movements are variable so that the motor system can discover the optimal pattern of muscle activation. A recent study that used theta-burst TMS as intervention before motor learning suggested that improved performance on the thumb abduction task might occur by two mechanisms: either increasing the variability of movement output or the stability of successful motor memories (Teo et al., 2011). Theta-burst TMS appeared to influence learning by increasing movement variability, which improved motor performance by increasing exploration of task-related levels of motor output (Teo et al., 2011). In the present task, sensory attention to vibration improved motor learning without increasing movement variability; in fact, variability decreased over time. We propose that attention may therefore have improved the sensorimotor memory of successful movements to make the learning process more efficient. Compared with theta-burst TMS, the sensory attention tasks had no effect on overall levels of cortical excitability since MEPs and SICI measured in the absence of muscle vibration were similar before and after AttVIB and AttCUT. Instead, our findings suggest the learning rates were affected via a specific effect of attention on the integration of muscle-specific proprioceptive input into motor cortex.

Figure 6 gives a hypothetical model of how this may have occurred. During performance of the motor learning task, the 
abduction movements of the thumb evoke proprioceptive feedback from the movement, mainly from the APB muscle that is most engaged in this task. Simultaneously, the system produces an internal estimate of the expected feedback based on the motor output responsible for moving the thumb. This is compared with the sensory information about the actual movement that was made. The result could either be used to improve the remembered estimate of the motor command for subsequent trials. Alternatively, it could be that, over time, an internal model of the task is constructed and in this case a sensory error signal could be used to optimize the model further.

It has been suggested that sensory attention can increase the behavioral impact of sensory inputs in several ways, such as by increasing the gain of the signal, reducing the noise in the incoming sensory signal, or promoting efficient selection of sensory inputs (Pestilli et al., 2011) [see discussion in the study by Serences (2011)]. We hypothesize that AttVIB engages similar processes with the consequence that sensory input from the movement can be used more efficiently to optimize and speed up learning. In contrast, AttCUT distracted subjects' attention from proprioceptive input. During the subsequent motor learning task, the integration of proprioceptive input might have even been impaired, which consequently led to deterioration in motor learning.

Given the short timescale of 15 min during which the sensory attention tasks induce these neurophysiological and behavioral changes, one contributing physiological mechanism may be an unmasking (for AttVIB) and masking (for AttCUT) of latent lateral intracortical connections (Jacobs and Donoghue, 1991) specific for proprioceptive-motor integration by changes in the activity of GABAergic inhibitory interneurons. Modifications in the level of GABA have been shown previously to play an important role in human motor learning (Ziemann et al., 2001; FloyerLea et al., 2006; Stagg et al., 2011) and, furthermore, to influence the formation of LTP (Komaki et al., 2007). The motor learning task we used here uses an LTP-like mechanism (Ziemann, 2004; Stefan et al., 2006; Rosenkranz et al., 2007). It is therefore possible that enhancing (for AttVIB) and reducing (for AttCUT) the efficacy of proprioception to reduce GABAergic intracortical inhibition leads to a facilitation or reduction, respectively, of use-dependent LTP-like synaptic plasticity.

The present findings are a striking mirror reversal of those of Ostry et al. (2010). They showed that motor learning affects sensory discrimination, whereas the present data show that sensory attention influences subsequent motor learning. Our study also extends their findings in two ways. First, the study by Ostry et al. involved movement performance, which necessarily involves both motor output as well as the sensory feedback that it generates. Thus, it is difficult to separate potential contributions of each to the process of motor learning. In contrast, our sensory attention tasks used muscle vibration, which produces a proprioceptive input that resembles afferent movement feedback, but in the absence of any motor output. Thus, there is strong evidence that the effect of these sensory attention tasks on subsequent motor learning is a true "cross-system" effect from the sensory to the motor system. Second, the sensory attention tasks used in our study involved performance but not learning of a sensory discrimination task. Indeed, the behavioral data showed no significant change in discrimination performance as the task progressed. Nevertheless, the tasks produced changes in sensorimotor integration of afferent input within the motor cortex. We speculate that, although there was no direct effect on discrimination performance, the attention to sensory input re- quired to perform the tasks had a direct influence on transmission of sensory input to motor cortex in a way that was important for subsequent motor learning.

In summary, this study shows for the first time that human motor learning can be shaped by selective modulation of proprioceptive integration in the motor cortex and, therefore, that changes in sensorimotor integration can drive changes in motor behavior. These findings are of particular relevance for the development of strategies to enhance the efficacy of learning (e.g., in motor skill learning) or to support functional recovery after brain injury or stroke in neurorehabilitation.

\section{References}

Alitto HJ, Usrey WM (2003) Corticothalamic feedback and sensory processing. Curr Opin Neurobiol 13:440-445.

Burke D, Hagbarth KE, Löfstedt L, Wallin BG (1976) The responses of human muscle spindle endings to vibration during isometric contraction. J Physiol 261:695-711.

Burton H, Sinclair RJ, McLaren DG (2008) Cortical network for vibrotactile attention: a fMRI study. Hum Brain Mapp 29:207-221.

Di Lazzaro V, Pilato F, Dileone M, Ranieri F, Ricci V, Profice P, Bria P, Tonali PA, Ziemann U (2006) $\mathrm{GABA}_{\mathrm{A}}$ receptor subtype specific enhancement of inhibition in human motor cortex. J Physiol 575:721-726.

Florian J, Müller-Dahlhaus M, Liu Y, Ziemann U (2008) Inhibitory circuits and the nature of their interactions in the human motor cortex a pharmacological TMS study. J Physiol 586:495-514.

Floyer-Lea A, Wylezinska M, Kincses T, Matthews PM (2006) Rapid modulation of GABA concentration in human sensorimotor cortex during motor learning. J Neurophysiol 95:1639-1644.

Fromm C, Wise SP, Evarts EV (1984) Sensory response properties of pyramidal tract neurons in the precentral motor cortex and postcentral gyrus of the rhesus monkey. Exp Brain Res 54:177-185.

Golaszewski SM, Siedentopf CM, Baldauf E, Koppelstaetter F, Eisner W, Unterrainer J, Guendisch GM, Mottaghy FM, Felber SR (2002) Functional magnetic resonance imaging of the human sensorimotor cortex using a novel vibrotactile stimulator. Neuroimage 17:421-430.

Hagbarth KE, Eklund G (1968) The effects of muscle vibration in spasticity, rigidity, and cerebellar disorders. J Neurol Neurosurg Psychiatry 31:207-213.

Heath CJ, Hore J, Phillips CG (1976) Inputs from low threshold muscle and cutaneous afferents of hand and forearm to areas 3a and 3b of baboon's cerebral cortex. J Physiol 257:199-227.

Hore J, Preston JB, Cheney PD (1976) Responses of cortical neurons (areas 3a and 4) to ramp stretch of hindlimb muscles in the baboon. J Neurophysiol 39:484-500.

Huffman KJ, Krubitzer L (2001) Thalamo-cortical connections of areas 3a and M1 in marmoset monkeys. J Comp Neurol 435:291-310.

Ilić TV, Meintzschel F, Cleff U, Ruge D, Kessler KR, Ziemann U (2002) Short-interval paired-pulse inhibition and facilitation of human motor cortex: the dimension of stimulus intensity. J Physiol 545:153-167.

Jacobs KM, Donoghue JP (1991) Reshaping the cortical motor map by unmasking latent intracortical connections. Science 251:944-947.

Jenkins WM, Merzenich MM, Ochs MT, Allard T, Guíc-Robles E (1990) Functional reorganization of primary somatosensory cortex in adult owl monkeys after behaviorally controlled tactile stimulation. J Neurophysiol 63:82-104.

Komaki A, Shahidi S, Lashgari R, Haghparast A, Malakouti SM, Noorbakhsh SM (2007) Effects of GABAergic inhibition on neocortical long-term potentiation in the chronically prepared rat. Neurosci Lett 422:181-186.

Kujirai T, Caramia MD, Rothwell JC, Day BL, Thompson PD, Ferbert A, Wroe S, Asselman P, Marsden CD (1993) Corticocortical inhibition in human motor cortex. J Physiol 471:501-519.

Marsden CD, Meadows JC, Hodgson HJ (1969) Observations on the reflex response to muscle vibration in man and its voluntary control. Brain 92:829-846.

Monfils MH, Plautz EJ, Kleim JA (2005) In search of the motor engram: motor map plasticity as a mechanism for encoding motor experience. Neuroscientist 11:471-483

Muellbacher W, Ziemann U, Wissel J, Dang N, Kofler M, Facchini S, Boroojerdi B, Poewe W, Hallett M (2002) Early consolidation in human primary motor cortex. Nature 415:640-644.

Ostry DJ, Darainy M, Mattar AA, Wong J, Gribble PL (2010) Somatosensory plasticity and motor learning. J Neurosci 30:5384-5393. 
Pavlides C, Miyashita E, Asanuma H (1993) Projection from the sensory to the motor cortex is important in learning motor skills in the monkey. J Neurophysiol 70:733-741.

Pestilli F, Carrasco M, Heeger DJ, Gardner JL (2011) Attentional enhancement via selection and pooling of early sensory responses in human visual cortex. Neuron 72:832-846.

Rioult-Pedotti MS, Friedman D, Hess G, Donoghue JP (1998) Strengthening of horizontal cortical connections following skill learning. Nat Neurosci 1:230-234.

Roll JP, Vedel JP, Ribot E (1989) Alteration of proprioceptive messages induced by tendon vibration in man: a microneurographic study. Exp Brain Res 76:213-222.

Rosenkranz K, Rothwell JC (2003) Differential effect of muscle vibration on intracortical inhibitory circuits in humans. J Physiol 551:649-660.

Rosenkranz K, Rothwell JC (2006a) Differences between the effects of three plasticity inducing protocols on the organization of the human motor cortex. Eur J Neurosci 23:822-829.

Rosenkranz K, Rothwell JC (2006b) Spatial attention affects sensorimotor reorganisation in human motor cortex. Exp Brain Res 170:97-108.

Rosenkranz K, Kacar A, Rothwell JC (2007) Differential modulation of motor cortical plasticity and excitability in early and late phases of human motor learning. J Neurosci 27:12058-12066.
Serences JT (2011) Mechanisms of selective attention: response enhancement, noise reduction, and efficient pooling of sensory responses. Neuron 72:685-687.

Stagg CJ, Bachtiar V, Johansen-Berg H (2011) The role of GABA in human motor learning. Curr Biol 21:480-484.

Stefan K, Wycislo M, Gentner R, Schramm A, Naumann M, Reiners K, Classen J (2006) Temporary occlusion of associative motor cortical plasticity by prior dynamic motor training. Cereb Cortex 16:376-385.

Teo JT, Swayne OB, Cheeran B, Greenwood RJ, Rothwell JC (2011) Human theta burst stimulation enhances subsequent motor learning and increases performance variability. Cereb Cortex 21:1627-1638.

Wong JD, Wilson ET, Gribble PL (2011) Spatially selective enhancement of proprioceptive acuity following motor learning. J Neurophysiol 105: 2512-2521.

Xerri C (2008) Imprinting of idiosyncratic experience in cortical sensory maps: neural substrates of representational remodeling and correlative perceptual changes. Behav Brain Res 192:26-41.

Ziemann U (2004) LTP-like plasticity in human motor cortex. Suppl Clin Neurophysiol 57:702-707.

Ziemann U, Lönnecker S, Steinhoff BJ, Paulus W (1996) The effect of lorazepam on the motor cortical excitability in man. Exp Brain Res 109:127-135.

Ziemann U, Muellbacher W, Hallett M, Cohen LG (2001) Modulation of practice-dependent plasticity in human motor cortex. Brain 124:11711181. 